IOS Press

\title{
Thesis
}

\section{User study and integration of assistive technologies for people with cognitive disabilities in their daily life activities}

\author{
Javier Gomez * and PhD Advisor Germán Montoro \\ Computer Engineering Department, Universidad Autónoma de Madrid, 28049, Madrid, Spain
}

\begin{abstract}
The present article summarizes the doctoral dissertation of Javier Gomez.
Keywords: Assistive technologies, cognitive disabilities, mobile computing, human-computer interaction
\end{abstract}

On May, 8th, 2015, the author defended his PhD thesis entitled "User study and integration of assistive technologies for people with cognitive disabilities in their daily life activities". Prof. Dr. Germán Montoro was the doctoral advisor. The viva took place at the Polytechnic School of the Autonomous University of Madrid, Spain, where the review committee included Prof. Dr. Xavier Alamán, Prof. Dr. Rosa M. Carro, Prof. Dr. Juan Carlos Augusto, Prof. Dr. Ramón López-Cózar and Prof. Dr. Javier Bajo. The earned degree was awarded with honours.

\section{Thesis summary}

New technologies are present in almost all activities of our lives, either in the work place (e.g. computers) or our leisure time (3D digital movie theatres, ebooks or videogames). Besides, we are unconsciously carrying a significant amount of computing and communication capabilities with us: our smartphones. In the last years, thanks to the improvements in their hardware and software, the number and variability of tasks that can be performed with these devices is growing up to

\footnotetext{
*Corresponding author. E-mail: jg.escribano@uam.es.
}

a level beyond phone calls and short-text messaging. Current devices allow us to access the Internet through wide-band connections, checking the email or social networks, playing multimedia content and many other tasks that used to be restricted to computers. Moreover, all of these actions can be carried out ubiquitously, regardless our location, thanks to the mobility they offer.

Apart from productivity or digital leisure activities, in the last years the interest on the application of these technologies to help people with special needs has increased. These developments, either focused on smartphones or any other device, are commonly tagged as "Assistive Technologies". The interest of the scientific community relies on the theoretical results and technical developments, but also on the high impact that these works may cause on the society. Thus, thanks in part to the research done in this area, people with disabilities are able to live more independent and autonomously. And even, access to new opportunities, such as getting a job.

This thesis is focused on the application of smartphones as assistive devices to help people with cognitive disabilities in their daily life activities. Particularly, it presents the "AssisT" project. This approach arises to address the need of integrated assistive systems, this is, systems of support that cover all the 


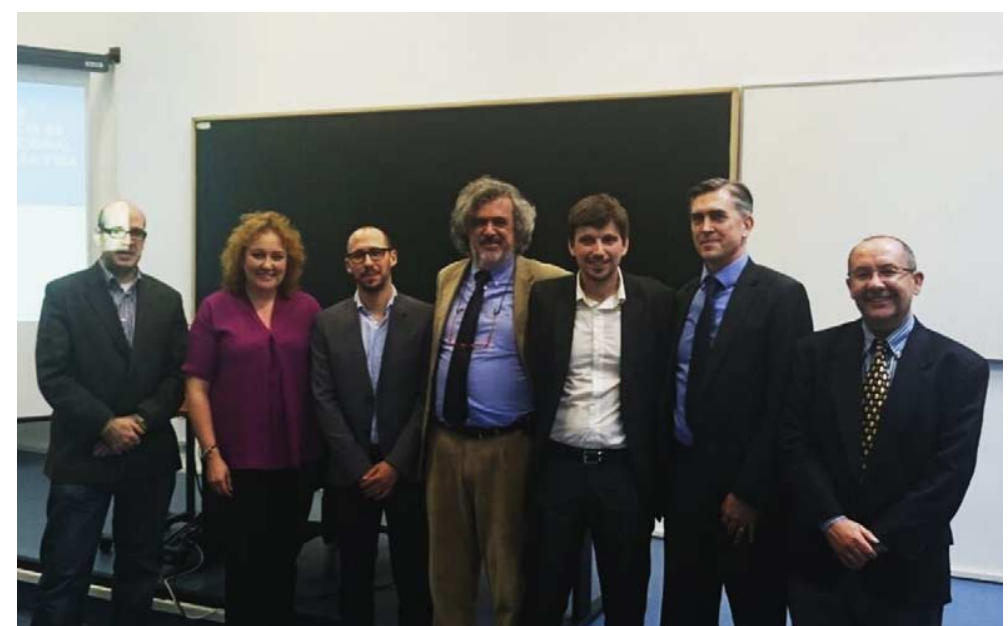

Fig. 1. PhD oral defense. From the left to the right: Dr. Javier Bajo, Dr. Rosa M. Carro, Dr. Javier Gomez, Dr. Xavier Alamán, Dr. Germán Montoro, Dr. Juan C. Augusto and Dr. Ramón López-Cózar.

users' activities during the day. Besides, it has been designed to adapt to users' needs, the activity and their context. To do that, the system is composed of three modules: "AssisT-Task” [1], "AssisT-Out” [2] and "AssisT-In" [3], which provide assistance on instrumental activities, navigation outdoors and navigation indoors, respectively.

The evaluation and development processes have followed a user centred design. To do that, experts from education and care of people with cognitive disabilities have participated actively. During the evaluation of the different modules a total of 206 sessions took place, in which 45 users with cognitive disabilities participated. The results extracted from the evaluation processes were very positive. Therefore, the different solutions and approaches have been validated. Thus, AssisT can be considered as a feasible and functional integrated assistive system, setting the design bases and improvements for future developments.

\section{References}

[1] J. Gomez, G. Montoro, P.A. Haya, X. Alamán, S. Alves and M. Martínez, Adaptive manuals as assistive technology to support and train people with acquired brain injury in their daily life activities, Personal and Ubiquitous Computing 17(6) (2013), 1117-1126.

[2] J. Gomez and G. Montoro, Design considerations and evaluation methodology for adapted navigational assistants for people with cognitive disabilities, in: 8th International Conference on Health Informatics, HEALTHINF, 2015, pp. 344-352.

[3] J. Gomez, X. Alamán, G. Montoro, J.C. Torrado and A. Plaza, AmICog - mobile technologies to assist people with cognitive disabilities in the work place, ADCAIJ: Advances in Distributed Computing and Artificial Intelligence Journal 2(7) (2014), 917. 